\title{
High Resolution Temperature Insensitive Interrogation Technique for FBG Sensors
}

\author{
Qiang Wu \\ Technological University Dublin, qiang.wu@tudublin.ie \\ Yuliya Semenova \\ Technological University Dublin, yuliya.semenova@tudublin.ie \\ An Sun \\ Technological University Dublin
}

See next page for additional authors

Follow this and additional works at: https://arrow.tudublin.ie/engscheceart

Part of the Electrical and Computer Engineering Commons

\section{Recommended Citation \\ Wu, Q. et al (2009) High Resolution Temperature Insensitive Interrogation Technique for FBG Sensors. Optics and Laser Technology, vol. 42, no. 4, p. 653-656. doi:10.1016/j.optlastec.2009.11.005}

This Article is brought to you for free and open access by the School of Electrical and Electronic Engineering at ARROW@TU Dublin. It has been accepted for inclusion in Articles by an authorized administrator of ARROW@TU Dublin. For more information, please contact arrow.admin@tudublin.ie, aisling.coyne@tudublin.ie, gerard.connolly@tudublin.ie.

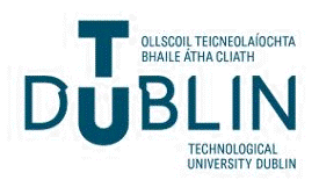


Authors

Qiang Wu, Yuliya Semenova, An Sun, Pengfei Wang, and Gerald Farrell

This article is available at ARROW@TU Dublin: https://arrow.tudublin.ie/engscheceart/66 


\title{
High resolution temperature insensitive interrogation technique for FBG sensors
}

\author{
Qiang Wu, Yuliya Semenova, An Sun, Pengfei Wang and Gerald Farrell \\ Photonics Research Centre, School of Electronic and Communications Engineering, \\ Dublin Institute of Technology, Kevin Street, Dublin 8, Ireland
}

\begin{abstract}
In this letter, we propose a high resolution temperature insensitive interrogation technique for FBG sensors where one FBG acts as an edge filter to interrogate a separate FBG sensor. A high resolution of better than $5 \mu \varepsilon$ in strain measurement range from 0 to $1100 \mu \varepsilon$ and the best resolution of better than $1 \mu \varepsilon$ were verified by experiments. An error of only $\pm 2.2 \mu \varepsilon$ is achieved over a temperature range from 15 to $50{ }^{\circ} \mathrm{C}$, indicating that this strain interrogation technique is temperature insensitive. Using an altered system configuration, the temperature was also measured simultaneously with a resolution better than $0.2^{\circ} \mathrm{C}$.
\end{abstract}

Index Terms—-Ratiometric system, FBG, Edge filter

\section{INTRODUCTION}

$\mathbf{F}$ ibre Bragg grating (FBG) based optical sensing technology has been extensively investigated and developed since the first FBG was demonstrated by Hill et al [1-18]. In many applications, the FBG sensor exists in a variable temperature environment. The change in temperature will lead to a centre wavelength shift of the FBG sensor, and hence will lead to measurement errors if the influence of temperature is not taken account of. The conventional way to account for the influence of temperature is to use an additional FBG sensor in the same environment to extract temperature information and hence the other information such as strain, pressure or force can be accurately extracted, with minimal temperature induced errors. The normal way to interrogate two or more FBG sensors is to use an optical spectra analyzer (OSA) or a scanning F-P filter to extract wavelength shifts [19-20]. This makes the interrogation system either high cost or complex. Other interrogation techniques for simultaneously extracting strain and temperature of FBG sensors have been developed [21-22]. However these techniques suffer the same disadvantages of high cost and complexity. Ratiometric wavelength measurement is a simple, high speed and cost effective scheme [23-24]. Recently Miao et al proposed a dynamic temperature compensated interrogation technique by using a tilted FBG as an edge filter in a ratiometric system [25]. This could reduce the complexity of the interrogation system, but the technique suffers at least three disadvantages: (1) the tilted FBG functioning as an edge filter, must be immersed in an index matching gel to get a smooth response - the process for embedding a tilted FBG with an index matching gel in a structure will be more complex compared to conventional embedding of FBG sensors; (2) the refractive index of the index matching gel will change if it exists in an ambient environment for a long time and hence the spectral response of the tilted FBG will change resulting in measurement errors and (3) the ambient temperature cannot be independently measured in such a system configuration.

In this paper, we propose a temperature insensitive interrogation technique for FBG sensors by utilizing two FBGs in a ratiometric system: one FBG functions as an edge filter and one narrow bandwidth FBG functions as a FBG strain sensor. Since both FBGs have similar wavelength shifts for the same temperature change, the measured ratio will be a constant if only the environmental temperature changes. However if strain is applied to the narrow bandwidth FBG sensor, the measured system ratio will change accordingly. Overall this makes the interrogation system temperature insensitive. Moreover an additional edge filter can be connected to the temperature compensating FBG to independently extract temperature information, if required and thus will realize simultaneous measurement both temperature and strain.

\section{SYSTEM DESCRIPTION}

A schematic diagram of the interrogation system is shown in Fig. 1.

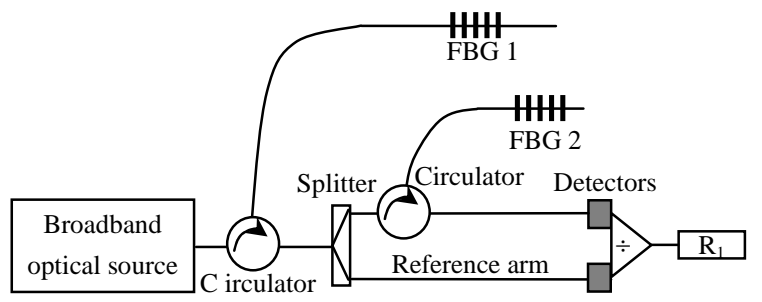

Fig. 1 Schematic diagram of the temperature insensitive ratiometric interrogation system

In Fig. 1, FBG 1 functions as an edge filter for FBG 2 and which is also used to compensate temperature induced wavelength shift, while FBG 2 functions as a strain sensor. The broadband optical source passes through FBG 1 first, and then was split into two paths: one goes to the FBG 2 and detected by a photodiode; the other one goes directly to a photodiode acting as a reference arm. The measured ratio $R_{1}$ is related to the strain. 
The normalized spectra of both FBG 1 and FBG 2 are shown in Fig. 2.

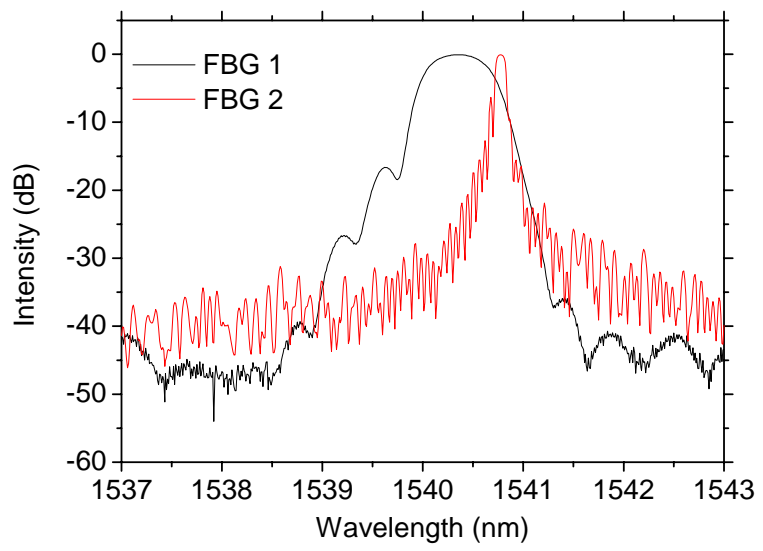

Fig. 2 Spectral response of two FBGs

In Fig. 2, the long wavelength edge of the reflected spectral response of the FBG 1 is used an edge filter and FBG 2 functions as a conventional FBG stain sensor. As temperature changes, the spectra of both FBG 1 and FBG 2 have similar wavelength shifts and hence the relative wavelength shift between them is very small. In this case the level of optical power reflected from the FBG 1 and passed through the splitter and reflected from the FBG 2 should remain the same and hence the measured ratio will remain constant when temperature change occurs. When strain is applied to FBG 2, only FBG 2 will show a wavelength shift and hence the reflected power level by FBG 2 will change. The strain can be easily extracted by monitoring the ratio variations.

Assuming the optical power density of the broadband optical source is 1 , the ratio $R_{1}$ of the system can be expressed as

$$
R_{1}=10 \log _{10}\left[\frac{\int G_{1 \lambda_{1}}(\lambda) G_{2 \lambda_{2}}(\lambda) d \lambda}{\int G_{1 \lambda_{1}}(\lambda) d \lambda}\right]
$$

Where $G_{1 \lambda_{1}}(\lambda)$ and $\lambda_{1}$ are the transmission response and the centre wavelength of FBG 1 respectively, while $G_{2 \lambda_{2}}(\lambda)$ and $\lambda_{2}$ are the transmission response and the centre wavelength of FBG 2 respectively.

Assuming the initial centre wavelengths of FBG 1and FBG 2 are $\lambda_{10}$ and $\lambda_{20}$, and the temperature and strain induced centre wavelength shifts are $\Delta \lambda_{t}$ and $\Delta \lambda_{s}$ respectively, Eq. (1) can be expressed as

$$
R_{1}=10 \log _{10}\left[\frac{\int G_{1 \lambda_{10}+\Delta \lambda_{t}}(\lambda) G_{2 \lambda_{20}+\Delta \lambda_{t}+\Delta \lambda_{s}}(\lambda) d \lambda}{\int G_{1 \lambda_{10}+\Delta \lambda_{t}}(\lambda) d \lambda}\right]
$$

For FBGs, once the temperature changes, the central wavelength of the reflected spectrum will shift, however the shape of the spectrum won't change. In this case the integral of spectrum remains unchanged and we have

$$
\int G_{1 \lambda_{10}}(\lambda) d \lambda \approx \int G_{1 \lambda_{10}+\Delta \lambda_{t}}(\lambda) d \lambda
$$

$$
\int G_{1 \lambda_{10}+\Delta \lambda_{t}}(\lambda) G_{2 \lambda_{20}+\Delta \lambda_{t}+\Delta \lambda_{s}}(\lambda) d \lambda \approx \int G_{1 \lambda_{10}}(\lambda) G_{2 \lambda_{20}+\Delta \lambda_{s}}(\lambda) d \lambda
$$

Eq. (2) can be rewritten as

$$
R_{1}=10 \log _{10}\left[\frac{\int G_{1 \lambda_{10}}(\lambda) G_{2 \lambda_{20}+\Delta \lambda_{s}}(\lambda) d \lambda}{\int G_{1 \lambda_{10}}(\lambda) d \lambda}\right]
$$

Eq. (5) contains no temperature induced wavelength shift term $\Delta \lambda_{t}$, so only strain induced wavelength shift $\Delta \lambda_{s}$, contributes to $R_{1}$, so that $R_{1}$ is temperature insensitive.

\section{EXPERIMENTAL RESULTS}

As an example, we carried out experimental investigations by applying strain to FBG 2 . The spectral responses of the two FBGs used in our experiments are shown in Fig. 2.

The relative wavelength shifts of the two FBGs vs. temperature, measured by an OSA with a resolution $0.01 \mathrm{~nm}$ is shown in Fig. 3(a-b).
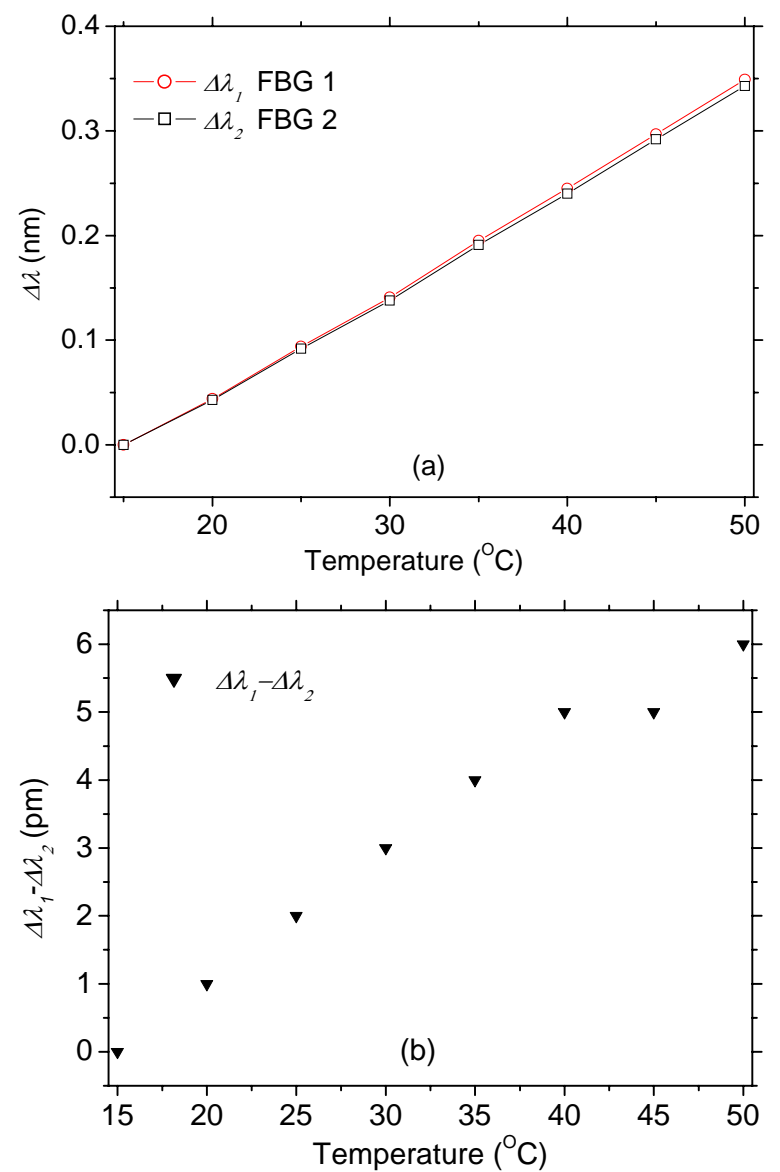

Fig. 3 Measured (a) wavelength shifts and (b) relative wavelength shifts of FBG 1 and FBG 2 vs. temperature

In Fig. 3(a), the square and circle are the measured temperature induced wavelength shifts of FBG 1 and FBG 2 respectively, and the inverse triangle in Fig. 3(b) is temperature induced relative wavelength shifts between FBG 1 and FBG 2 . It shows that both FBGs have similar wavelength shifts and the maximum difference is $6 \mathrm{pm}$ at temperature range from $15^{\circ} \mathrm{C}$ 
to $50{ }^{\circ} \mathrm{C}$. The discontinuity between $40{ }^{\circ} \mathrm{C}$ and $45^{\circ} \mathrm{C}$ is due to the limited resolution of the OSA which is $10 \mathrm{pm}$ and the measured temperature induced wavelength shifts difference between FBG 1 and FBG 2 is 6 pm.

Further experiments were carried out by applying strain to FBG 2 at room temperature. The measured ratio vs. strain was shown in Fig. 4 (bottom axis).

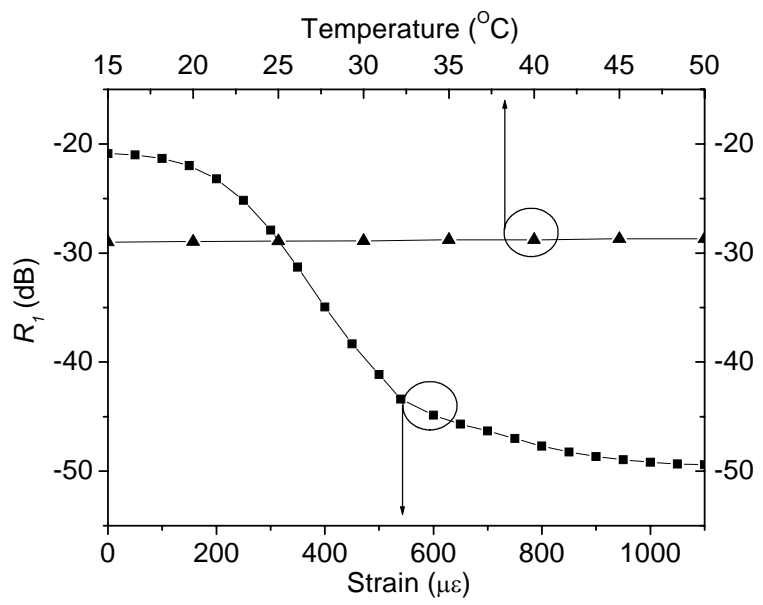

Fig. 4 Measured ratio $R_{1}$ vs. strain at room temperature and measured ratio $R_{1}$ vs. temperature at fixed strain

Figure 4 shows that as strain increases from 0 to $1100 \mu \varepsilon$, the measured ratio $R_{1}$ decreases monotonically and the rate of decrease (corresponding to the slope) increases firstly and then decreases. To investigate the influence of temperature on the system ratio variations, experiments were carried out by increasing the temperature from 15 to $50^{\circ} \mathrm{C}$ at a fixed strain of $320 \mu \varepsilon$. The ratio response of $R_{1}$ is shown in Fig. 4 (top axis).

Our calculations show that the ratio variation of $R_{1}$ is only $\pm 0.15 \mathrm{~dB}$ for temperature range from 15 to $50{ }^{\circ} \mathrm{C}$, which corresponds to strain variation of $\pm 2.2 \mu \varepsilon$ or $\pm 0.063 \mu \varepsilon /{ }^{\circ} \mathrm{C}$. This result indicates that the FBG strain sensor is temperature insensitive, which matches well with Eq. (5).

To illustrate the resolution of this system, an incremental step change of $50 \mu \varepsilon$ and $2 \mu \varepsilon$ are applied to the FBG 2 sensor. The measured ratio variations $R_{1}$ vs. time are shown in Fig. 5(a-b).

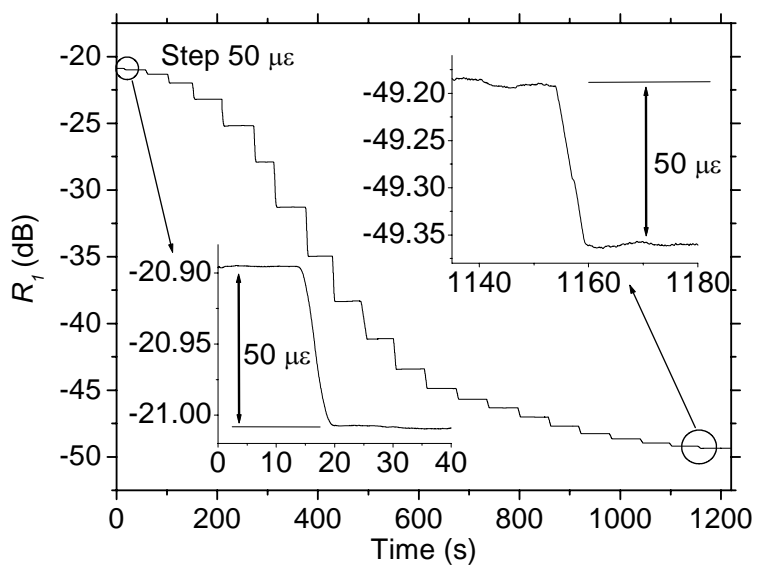

(a)

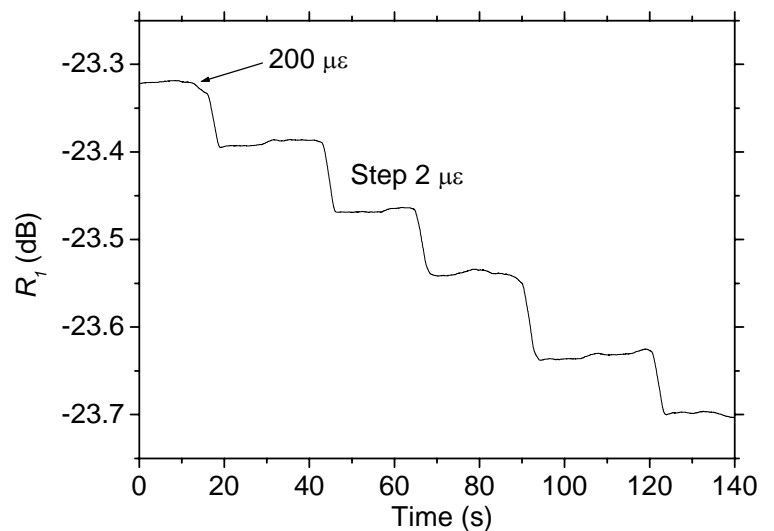

(b)

Fig. 5 Measured ratio $R_{1}$ variations vs. time with (a) step change $50 \mu \varepsilon$ and (b) step change of $2 \mu \varepsilon$ from $200 \mu \varepsilon$ to $220 \mu \varepsilon$

Figure 5(a) shows that there is minimum ratio change step at strain range from 0 to $50 \mu \varepsilon$ and from 1050 to $1100 \mu \varepsilon$. The maximum peak-to-peak ratio variation is $0.014 \mathrm{~dB}$ and the minimum discrimination over the whole strain range from 0 to $1100 \mu \varepsilon$ is $0.16 \mathrm{~dB}$ for a step change of $50 \mu \varepsilon$. Hence we can estimate the worst case resolution of this system as at least $5 \mu \varepsilon$ over the whole strain measurement range from 0 to $1100 \mu \varepsilon$. Figure 5(b) shows the effect of small strain step change of $2 \mu \varepsilon$ from 200 to $220 \mu \varepsilon$, which indicates that the resolution over certain strain ranges is better than $1 \mu \varepsilon$.

\section{ADAPTATION OF THE SYSTEM TO MEASURE TEMPERATURE AND STRAIN SIMULTANEOUSLY}

It has been shown that the system presented above can be used for measurement of strain with dynamic temperature compensation. If we want to measure both strain and temperature simultaneously, an additional edge filer can be added to the system to measure temperature as shown in Fig. 6.

In Fig. 6, FBG 1 functions as an edge filter which used to compensate temperature induced wavelength shift and FBG 2 functions as a strain sensor. The broadband optical source passes through FBG 1 first, and then was split into three paths: one goes to the FBG 2 and detected by a photodiode; one goes directly to a photodiode acting as a reference arm; the last one passes through a second edge filter (in this case it is a thin film edge filter, other types of edge filter could be used) and finally is detected by a photodiode. The measured ratios $R_{1}$ and $R_{2}$ are related to the strain and temperature respectively.

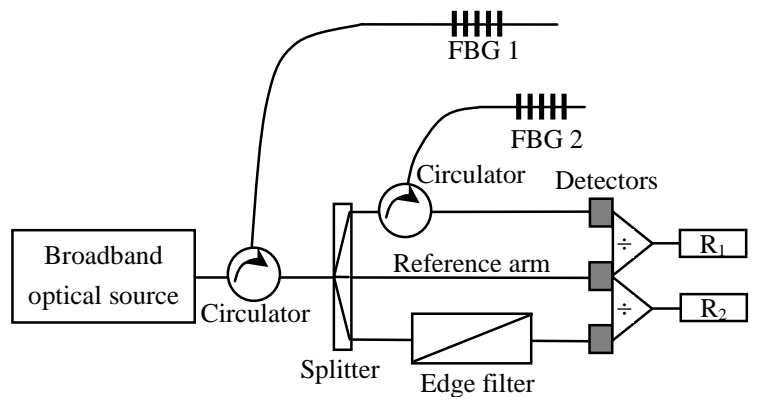

Fig. 6 Schematic diagram for simultaneous measurements of strain and temperature 
Since the system configuration is similar to that in Fig. 1, experiments were only carried out for $R_{2}$. Figure 7 shows the ratio change as the temperature increases from 15 to $50{ }^{\circ} \mathrm{C}$. It is noted that the curve is normalized to a common ratio value of 0 dB at $15{ }^{\circ} \mathrm{C}$.

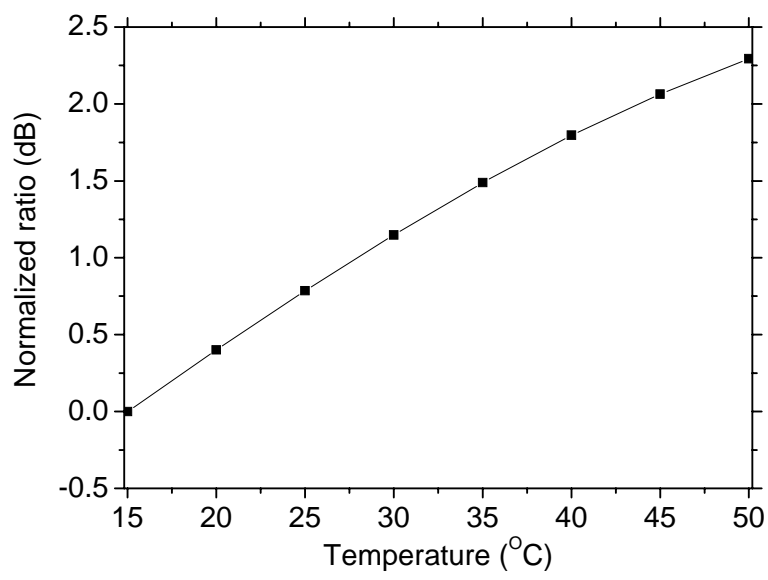

Fig. 7 Measured ratio $R_{2}$ variations vs. temperature

Figure 7 shows that as temperature increase from 15 to $50{ }^{\circ} \mathrm{C}$, the measured ratio of $R_{2}$ increases monotonically and can be used to extract temperature information. The discrimination from 15 to $50{ }^{\circ} \mathrm{C}$ is $2.29 \mathrm{~dB}$ and hence the average temperature ratio change is $0.065 \mathrm{~dB} /{ }^{\circ} \mathrm{C}$. Assuming the minimum resolution of the photodiode is $0.01 \mathrm{~dB}$, then the temperature resolution of this system is better than $0.2^{\circ} \mathrm{C}$.

\section{CONCLUSION}

A high resolution temperature insensitive interrogation technique for FBG sensors is proposed. This technique uses two FBGs: one functions as an edge filter to demodulate another FBG which functions as a strain sensor. Experimental results show that this technique has a high resolution of at least $5 \mu \varepsilon$ for strain measurement from 0 to $1100 \mu \varepsilon$ and that the best resolution can be better than $1 \mu \varepsilon$ for certain strain ranges. Experiments verified that this technique is temperature insensitive with only error of $\pm 0.063 \mu \varepsilon /{ }^{\circ} \mathrm{C}$ over a temperature range from 15 to $50^{\circ} \mathrm{C}$. By adding an additional edge filter and photodiode, the temperature can be measured simultaneously. Experimentally the resolution achieved is better than $0.2{ }^{\circ} \mathrm{C}$.

\section{REFERENCES}

[1]. K. O. Hill, Y. Fujii, D. C. Johnson, B. S. Kawasaki, "Photosensitivity in optical fiber waveguides - application to reflection filter fabrication", Applied physics letters, vol. 32, no. 10, pp. 647-649, 1978

[2]. A. B. Lobo Ribeiro, L. A. Ferreira, M. Tsvetkov, J. L. Santos, “All-fiber interrogation for fiber Bragg sensors using a biconical fiber filter", Electronics Letters, vol. 6, no. 6, pp. 757-759, 1994

[3]. R. W. Fallon, L. Zhang, L. A. Everall, J. A. R. Williams, I. Bennion, “All-fiber optical sensing system: Bragg grating sensor interrogated by a long-period grating”, Measurement Science and Technology, vol. 9, no. 12, pp. 1969-1973, 1998

[4]. M. A. Davies, A. D. Kersey, "All-fiber Bragg grating strain-sensor demodulation technique using a wavelength division coupler”, Electronics Letters, vol. 30, no. 1, pp. 75-76, 1994

[5]. S. Chung, J. Kim, B. A. Yu, B. Lee, “A fiber Bragg grating sensor demodulation technique using a polarization maintaining fiber loop mirror”, Photonics Technology Letters, vol. 13, no. 12, pp. 1343-1345, 2001

[6]. L. A. Ferreira, J. L. Santos, "Demodulation scheme for fiber Bragg sensors based on source spectral characteristics”, Pure Applied Optics vol. 5, pp. 257-261, 1996

[7]. D. A. Jackson, A. B. Lobo Ribeiro, L. Reekie, J. L. Archambault, "Simple multiplexing scheme for a fiber-optic grating sensor network", Optics Letters vol. 18, no. 14, pp. 1192-1194, 1993

[8]. Qiang Wu, Agus Muhammad Hatta, Yuliya Semenova and Gerald Farrell, "Use of a SMS fiber filter for interrogating FBG strain sensors with dynamic temperature compensation”, Applied Optics, vol. 48, pp. 5451-5458, 2009

[9]. R. Romero, O. Frazão, P. V. S. Marques, H. M. Salgado, J. L. Santos, "Fiber Bragg grating interrogation technique based on a chirped grating written in an erbium-doped fiber”, Measurement Science and Technology, vol. 14, pp. 1993-1997, 2003

[10]. A. B. Lobo Ribeiro, L. A. Ferreira, J. L. Santos, D. A. Jackson. "Analysis of the reflective-matched fiber Bragg grating sensing interrogation scheme”,Applied Optics,vol. 36, no. 4, pp. 934-939, 1997

[11]. L. A. Ferreira, J. L. Santos, F. Farahi, "Pseudo-heterodyne demodulation technique for fiber Bragg grating sensors using two matched gratings”, Photonics Technology Letters, vol. 9, no. 4, pp. 487-489, 1997

[12]. S. C. Kang, S. Y. Kim, S. B. Lee, S. W. Kwon, S. S. Choi, B. Lee, "Temperature-independent strain sensor system using a tilted Bragg grating demodulator”, Photonics Technology Letters, vol. 10, no. 10, pp. 1461-1463, 1998

[13]. D. Zhao, X. Shu, L. Zhang, I. Bennion, "Sensor interrogation technique using chirped fiber grating based Sagnac loop”, Electronics Letters, vo. 38, no. 7, pp. 312-313, 2002

[14]. C. Jáuregui, A. Quintela, J. M. López-Higuera, "Interrogation unit for fiber Bragg grating sensors that uses a slanted fiber grating”, Optics Letters, vol. 29, no. 7, pp. 676-678, 2004

[15]. J. S. Wu, S. L. Tsao, "Highly accurate temperature sensor using two fiber Bragg gratings”, US Patent 6024488

[16]. A. Cusano, G. Breglio, M. Giordano, L. Nicolais, "Low-cost all-fiber Bragg grating sensing system for temperature and strain measurements”, Optical Engineering, Vol. 44, no. 8, pp. 084402, 2005

[17]. Y. B. Lin, C. L. Pan, Y. H. Kuo, K. C. Chang and J. C. Chern, "Online monitoring of highway bridge construction using fiber Bragg grating sensors”, Smart Materials \& Structures, vol. 14, no. 5, pp. 1075-1082, Oct. 2005

[18]. A. Hongo, S. Kojima and S. Komatsuzaki, “Applications of fiber Bragg grating sensors and high-speed interrogation techniques”, Structural Control \& Health Monitoring, vol. 12, no. 3-4, pp. 269-282, Jul.-Dec. 2005

[19]. A. D. Kersey, T. A. Berkoff, W. W. Morsey, "Multiplexed fiber Bragg grating strain-sensor system with a fiber Fabry-Perot wavelength filter", Optics Letters, vol. 18, no. 16, pp. 1370-1372, Aug. 1993

[20]. M. A. Davis, D. G. Bellemore and A. D. Kersey, "Structural stain mapping using a wavelength/time division addressed fiber Bragg grating array”, in Proc. SPIE 1994 2nd European Conf. Smart Struct. Materials, Glasgow, Scotland, Oct. 1994, vol. 2361, pp. 342-345

[21]. L. V. Nguyen, D. Hwang, D. S. Moon, Y. Chung, "Simultaneous measurement of temperature and strain using a Lyot fiber filter incorporated with a fiber Bragg grating in a linear configuration”, Measurement Science \& Technology, vol. 20, no. 3, Article Number: 034006, March 2009

[22]. L. Y. Shao, X. Y. Dong, A. P. Zhang, H. Y. Tam and S. L. He, "High-resolution strain and temperature sensor based on distributed Bragg reflector fiber laser”, IEEE Photonics Technology Letters, vol. 19, no. 17-20, pp. 1598-1600, Sep.-Oct. 2007

[23]. S. M. Melle, K. Liu and R. M. Measures, “A passive wavelength demodulation system for guided-wave Bragg grating sensors”, IEEE Photonics Technology Letters, vol. 4, no. 5, pp. 516-518, May 1992

[24]. M. A. Davis, A. D. Kersey, "Matched-filter interrogation technique for fiber Bragg grating arrays”, IEE Electronics Letters, vol. 31, no. 10, pp. 822-823, May 1995

[25]. Y. P. Miao, B. Liu, W. H. Zhang, B. Dong, H. B. Zhou and Q. D. Zhao, "Dynamic temperature compensating interrogation technique for strain sensors with tilted fiber Bragg gratings”, IEEE Photonics Technology Letters, vol. 20, no. 6, pp. 1393-1395, Aug. 2008 